\title{
Formación de valores sociales desde el enfoque de convivencia pacífica ${ }^{1}$ \\ Formation of social values from the peaceful coexistence approach
}

DOI: http://dx.doi.org/10.17981/cultedusoc.9.3.2018.15

Artículo de investigación. Fecha de recepción: 15/06/2018. Fecha de aceptación: 27/11/2018

\author{
Aileen Cadena ${ }^{2}$; \\ Celina Arango; Peggy Luqueta; Eblin Ospina; Emelith Rodríguez; \\ Gladys Jiménez; Jackeline Vilardy y Nubiola Guardo3 \\ IED Arcesio Caliz Amador, Sede Principal (Colombia) \\ ailcadena@gmail.com
}

Para citar este artículo:

Cadena, A., Arango, C., Luqueta, P., Ospina, E., Rodríguez, E., Jiménez, G., Vilardy, J. y Guardo, N. (2018). Formación de valores sociales desde el enfoque de convivencia pacífica. Cultura. Educación y Sociedad 9(3), 123-130. DOI: http://dx.doi.org/10.17981/cultedusoc.9.3.2018.15

\section{Resumen}

Los valores sociales son aquellos que permiten a los individuos convivir en armonía, aceptando diferencias y políticas socialmente establecidas. El presente estudio tiene como objetivo identificar la formación de valores sociales que tiene los estudiantes de la institución educativa Arcesio Cáliz Amador Para la promulgación de la convivencia pacífica, la autoconciencia, a través de estrategias lúdico - pedagógica. Este estudio se llevará a cabo desde una mirada Cualitativa, soportado en el paradigma histórico hermenéutico, con un alcance Etnográfico Descriptivo. La Población participante son los estudiantes de Grado $8^{\mathrm{a}}$ y $8 \mathrm{~b}$, una muestra de 25 estudiantes. Como resultados se encontró que inicialmente, los estudiantes no tenían claro el concepto de valores sociales, no obstantes si logran definir que es el respeto, tolerancia, amor, entre otros. De igual manera ponen en práctica algunos valores, pero se debe seguir potenciando para aumentar la sana convivencia.

Palabras clave: Valores sociales; convivencia pacífica; autoconciencia; estrategias lúdico- pedagógicas.

\section{Abstract}

Social values are those that allow individuals to coexist in harmony, accepting socially established differences and policies. This study aims to identify the formation of social values that have students from the school Arcesius Chalice Amador for the enactment of peaceful coexistence, selfawareness through playful strategies - teaching. This study will be carried out from a Qualitative perspective, supported in the historical hermeneutical paradigm, with a Descriptive Ethnographic scope. The participating population is the 8th and 8th grade students, a sample of 25 students. As results were found that initially, the students were not clear about the concept of social values, not obstructing if they manage to define what is respect, tolerance, love, among others. In the same way, they put some values into practice, but they must continue to be potentiated in order to increase healthy coexistence.

Keywords: Social values; peaceful coexistence; selfawareness; playful-pedagogical strategies.

\footnotetext{
1 Este artículo ha sido derivado del Proyecto Fortalecimiento de la Cultura Ciudadana y Democrática en CT+I a través de la IEP apoyada en TIC en el Dpto. del Magdalena. Ciclón.

2 Líder del grupo de investigación "Forjadores de paz y convivencia”.

3 Docentes pertenecientes al grupo de investigación "Forjadores de paz y convivencia” de la IED Arcesio Caliz Amador, sede Principal. Ciclón Magdalena, estrategia $N^{\circ} 2$. Universidad de la Costa CUC..
}

- The author; licensee Universidad de la Costa - CUC. 


\section{Introducción}

La realidad de las sociedades resulta compleja y desequilibrada, y es el ejercicio del derecho el que se permite validar a partir del proceso interno que se alimentan dentro de los sistemas sociales para lograr una adaptación y adecuada realización de su función en términos de justicia social, y que se promulgan a la luz de los valores sociales (Herrera, Certain y Calderón. 2014).

En la actualidad el país ha centrado sus esfuerzos en la consecución de la paz estable y duradera, las instituciones educativas no han sido ajenas a este proceso y desde los entornos escolares se ha contribuido a la disminución de los conflictos, la convivencia pacífica es sinónimo de paz.

Cruz (2016) expone que Colombia siempre ha sido una excepción en términos de las dinámicas de los procesos políticos latinoamericanos, los actores sociales y políticos poco a poco se han ido permeando de eso que podríamos denominar "revolución semántica", que ha caracterizado los procesos de transformación, con todo y sus limitaciones, en otros países de la región (p. 54)

Hay opiniones muy diversas en cuanto a lo que es la Educación para la Paz. Según la UNESCO, es el "proceso de promoción de conocimientos, habilidades, actitudes y valores necesarios para lograr cambios de comportamiento que permitan a niños y niñas, jóvenes y adultos prevenir los conflictos y la violencia, tanto manifiestos como estructurales, resolver los conflictos pacíficamente y crear las condiciones propicias para la paz, ya sea en un nivel intrapersonal, interpersonal, intergrupal, nacional o internacional" (UNESCO, 2011).

Según Vásquez (2010),

la educación para la paz tiene muchos significados divergentes para diferentes individuos en diferentes lugares. Para algunos, la educación para la paz es principalmente un asunto de cambio de mentalidad; el propósito general es promover comprensión, respeto y tolerancia frente a los que eran enemigos en el pasado" (2010).
Para otros, la temática integralmente debe tener en cuenta las habilidades desde la asunción que la violencia no es el derrotero para la resolución de conflictos (Arrieta, 2016). Para los países del tercer mundo en particular, la educación para la paz está estrechamente vinculada a los derechos humano, mientras que, para otros, la mirada incluye otros sectores (Clemente, 2015).

Motivar la formación de valores sociales desde un enfoque de la convivencia pacífica que contribuya a la consecución de la paz es uno de los propósitos de la institución Arcesio Cáliz Amador que permita una relación de respeto, tolerancia, aceptación de las diferencias donde prime el dialogo para la mediación de los conflictos, se busca la participación directa y activa del estudiante, docente y comunidad en General logrando que todos los involucrados tomen conciencia de la importancia de la convivencia pacífica para lograr la paz.

En los últimos tiempos, se ha notado la creciente preocupación sobre la manera en cómo se están llevando las relaciones al interior de las Instituciones Educativas. Es entonces, la intolerancia, ausencia de respeto, maneras desviadas de resolver los conflictos los detonantes de inadecuada convivencia.

Esto se da debido al tronco de legislación de cada país y las limitaciones que se tienen para crecer, así como lo Expresa Cruz (2016)

que, en efecto, además de las limitaciones formales que el sistema electoral puede establecer para el reconocimiento de partidos, también la competencia por los votos presenta constricciones, como el alto costo de las campañas promovido por la inserción de la criminalidad organizada y los actores armados ilegales, en especial del paramilitarismo (p. 55)

En el mundo el estudio sobre violencia se llevó a cabo inicialmente en países como Noruega y Suecia, desde los años 1960 y 1980. Con estas investigaciones se resaltó 
e hizo un llamado a reflexionar las diferentes manifestaciones que se estaban presentando en las aulas, así mismo se tuvo en cuentas variables como género, estrato socio económico, los roles etc. Así mismo en países como En Australia se llevaron a cabo investigaciones que permitían distinguir los tipos de maltrato, desde los que resultan malos y los que no, partiendo del reconocimiento del agresor, el agredido y los demás participantes (Hurtado, 2008).

En la actualidad los educandos viven ciertas situaciones de violencia que se reflejan desde el seno familiar y que podrán generar condiciones de violencia e intolerancia en los individuos, algunas posibles causas son: Niños mucho tiempo solos, Poca o inexistente autoridad en el hogar, Múltiples e incorrectos modelos de comportamientos, El deseo desbordado de sexo, Las drogas, El alcohol, Falta de una adecuada formación en valores (Sanchez, 2015).

Las anteriores causas se presentan en un alto porcentajes en los hogares de los educandos que de una u otra manera se reflejan en el contexto educativo, dado a la intolerancia de algunos estudiantes en la hora de descanso, se presentan agresiones físicas y verbales, los docentes deben vigilar los descansos para evitar y/o controlar estas situaciones, se violan los derechos entre pares, estas situaciones que en ocasiones genera un grupo de estudiantes en la hora de descanso influye para que la convivencia pacífica sea posible en la Institución Educativa Arcesio Cáliz Amador esta investigación busca las posibles causas que los motivan a actuar de esta forma de igual manera fundamentar los valores sociales que disminuyan esta problemática.

Según lo expuesto por UNESCO (2011). Las Instituciones educativas están llamadas a involucrarse en la cultura para la paz a través de una sana convivencia, especialmente en Latinoamérica, en donde las seguidas reformas traen consigo impacto en la vida de las personas, facilitando diversas formas de pensar y actuar, y dentro de ellas se resalta la poca tolerancia a la diferencia, ausencia de valores éticos y morales, la constante y agresiva competencia, la desigualdad, el poco respeto al medio ambiente etc.

Es la familia como primer agente socializador de valores quien tiene un papel determinante en la vida de los niños y niñas, pues en ella se sientan las bases necesarias para la generación de autonomía, el respeto a los demás y la participación social (Oliva, 2006).

Los padres tal como lo expresa Oliva, (2016) son "los facilitadores legítimos del tránsito del espacio íntimo de las relaciones familiares a las relaciones más amplias con la sociedad y la cultura a la que ellos pertenecen"; son ellos los que pueden reconocer los cambios que vivencian los hijos de acuerdo a las reacciones contextuales y culturales.

La Paz debe construirse en la cultura y en la estructura sociales, no sólo en la mente humana, dando lugar a demandas socioeducativas orientadas a desarrollar en los ciudadano un sistemas de valores distintos, un cambio de ideal del hombre materialista al hombre existencial y convivencia, así como la construcción un nuevo proceso de globalización que dé cuenta de la vida y no del dinero. La Escuela adquiere en este marco especial importancia, pues es en ella donde se forma al ciudadano del que se demandan mayores niveles de participación, compromiso, además de una capacidad reflexiva y crítica para ejercer la tarea de la nueva conformación de los espacios relacionales (Velazco y Mosquera, 2010).

La cultura de la paz y la convivencia constituye un elemento dinamizador de la realidad interactiva que se apoya en la escuela el cual constituye el espacio geográfico y social de formación de ciudadanía 
y de participación comunitaria donde se asume la actividad educativa. En síntesis, este lugar tiene innumerables y complejas interacciones. Ella debería contribuir con la socialización, adaptación de las personas a la paz y la convivencia escolar pacífica, y al proceso de reflexión y acción. Pero, en muchos casos se desarrollan situaciones que generan intimidación, malos tratos, intimidación, abusos y agresiones psicológicas (Velazco y Mosquera, 2010).

Agregan Hernández, Meléndez y Chumaceiro (2017); se debe generar la formación ciudadana y democrática desde una cultura de paz dirigida para individuos responsables, con los conocimientos y competencias necesarias que ejerzan la participación activa, reflexiva y de dialogo, para la resolución de conflictos de forma no violenta, acatando las normas de actuación de valores establecidos en cada sociedad. (p. 45)

Bajo este contexto, el escenario educativo debe promover un sistema de interacciones sinérgicas que posibilite la promoción de valores de tolerancia, justicia, solidaridad, respeto a los derechos humanos y la no discriminación cónsonos al desarrollo de una pedagogía que se despliegue en el ámbito de las relaciones humanas construidas sobre la plataforma de la cooperación y participación en función de aprehender la pluralidad de pensamientos de los otros en un mundo de símbolos y significantes comunicativos para alcanzar la cultura en paz y la convivencia ciudadana.

En ese orden de ideas Araque y Suarez (2017), hablan de un modelo social, que es inclusivo en el trato igualitario de todas las personas desde la valoración y respeto por las diferencias;

"Conviene subrayar que este modelo se encuentra mediado por los derechos humanos, y espera potenciar el respeto por la libertad, igualdad y la inserción social, basándose en la no exclusión, la otredad, la vida independiente y el diálogo, entre otros" (p. 103).

\section{Educación como derecho}

Son los seres humanos los autores de los derechos, por la razón que son poseedores de dignidad. Por consiguiente, la dignidad es considerada como el "derecho a tener derechos". Un derecho primordial es el de la educación, y a ser educado en derechos humanos.

El Art.26, inciso 2 de la DUDH que:

"La educación tendrá por objeto el pleno desarrollo de la personalidad humana y el fortalecimiento del respeto a los derechos humanos y a las libertades fundamentales; favorecerá la comprensión, la tolerancia y la amistad entre todas las naciones y todos los grupos étnicos o religiosos, y promoverá el desarrollo de las actividades de las Naciones Unidas para el mantenimiento de la paz" (Rodino 2012).

La educación como derecho permite a sus participantes o involucrados empoderarse y trasformar su realidad. Cuando se reconocen los derechos, se puede distinguir de que se debe favorecer y de que no, hasta donde llegar, de que disfrutar. De los derechos con los que cuentan los individuos es el de la educación está inmerso en todos los derechos de los que gozan los seres humanos (Ministerio de Educación Nacional. 2012).

Analizado lo que expresan los autores se puede afirmar que la convivencia pacífica está implícita en la educación (Ochoa, 2015), en los centros educativos no solo se debe instruir a las niñas, niños y jóvenes, sino también se debe fortalecer en los educandos los valores sociales que los conviertan en ciudadanos amantes del cumplimiento de las leyes y normas que acepten las diferencias para que puedan convivir pacíficamente.

Es importante entonces mencionar las actitudes, siendo estas las que juegan un papel primordial en la construcción de convivencia pacífica, pues tal como lo expresa Casadiego, Martínez, Riatiga y Lopez, (2015). Estas son reacciones de la gente 
hacia el concepto, o usando el lenguaje coloquial sus sentimientos, creencias, y disposiciones hacia la acción. El concepto puede ser la persona, grupo, evento, objeto o una abstracción. Es una disposición psíquica y nerviosa, organizada por la experiencia que ejerce una influencia orientadora y dinámica, sobre las relaciones del individuo frente a todos los objetos y síntomas con los que está relacionado"

Se habla entonces también del aprendizaje de habilidades, las cuales facilitan al individuo un positivo desarrollo de las relaciones interpersonales, durante sus experiencias, entendiéndose esta como "la capacidad de realizar determinadas tareas, o resolver determinados problemas", subrayando su valor pragmático. Todas estas experiencias son aprendidas por la experiencia y el entrenamiento, pero también dependen de la forma en la que la persona hace valor a sus derechos y necesidades (Sanchez, 2017).

Respecto a la relación del profesor con sus alumnos se puede señalar que tradicionalmente los maestros son el factor crucial de la educación en el aula; pues a través de su práctica pedagógica pueden generar una atmosfera, ordenada y orientada al aprendizaje.

Alvarez, (2011) señala, que lo que caracteriza la actuación de los docentes es la tensión, la inseguridad y la agresividad igualmente. En las observaciones en el aula de clases, es muy notorio comportamiento de estudiantes violentos. Así mismo agregan que el autoritarismo del docente en las aulas de clases impulsa a los estudiantes a proceder con agresión y hostilidad, y esto a su vez, genera un clima socio-emocional desfavorable para el adecuado desarrollo de los procesos de enseñanza y aprendizaje.

De acuerdo a la investigación realizada por Mujica, (2013) que expone diversos análisis con relación a las relaciones interpersonales que se ponen en plenaria en los planteles educativos, y el papel del maestro en los mismos.

Son las relaciones dentro de las aulas de clases que están envueltas de poca armonía entre los docentes con los estudiantes, pues la mayoría de los intercambios que se establecen entre ellos, están mediatizados por comportamiento y actitudes cargados de violencia y agresividad, emociones negativas y antivalores; además porque los docentes asumen posiciones tradicionales en relación

Es la escuela, uno de los escenarios donde se potencian las relaciones, pues en el contacto directo profesor - estudiante, se comparten sentimientos, valores, pensamientos, ideas, etc.

\section{Convivencia escolar}

Según López, (2014). Inicialmente el termino Convivencia es entendido como "la acción de convivir, esto no es más que el compartir constante con otra persona diferente a ti todos los días, es decir, es la hecho de vivir en compañía con otros individuos", la interacción constante se convierte entonces en un elemento indispensable dentro de las relaciones con vivenciales en donde debe primar la serenidad, el respeto y buscando una distancia con las peleas, agresiones discusiones entre otras acciones que atentan contra la sana convivencia.

Se puede definir la convivencia como el clima de interrelaciones que se produce en la institución escolar, y que comprende las relaciones entre todos los miembros de la comunidad educativa (estudiantes, profesores/as, personal administrativo y padres de familia).

Según Jacques Delors en un informe presentado en la UNESCO en (1997) estableció que uno de los cuatro pilares de la educación es el aprender a vivir juntos; allí resalta la importancia de la convivencia en el aula para la educación además de los aspectos cognoscitivos e intelectuales. 
Últimamente los medios de comunicación nos han dado a conocer la ocurrencia de problemas de convivencia en las escuelas con casos de violencia, acoso y mal trato de la que son objeto los estudiantes y hasta algunos maestros o docentes, nos pone de manifiesto el tipo de relaciones interpersonales que se vive en ella y también pone en tela de juicio la calidad de nuestro sistema educativo. La presencia de estos hechos es algo normal en cualquier escuela del mundo, del país y de la localidad (Lopez, 2014).

Es la convivencia pacífica la que entonces, se basa en la negociación de los conflictos que se presentan en cualquier contexto, y en el específico en el campo educativo el propósito fundamental es fortalecer las relaciones de los estudiantes. Para lograr la convivencia pacífica es necesario aprender a comunicarse dentro de los límites del principio de la transparencia, lo cual requiere el diálogo como parte de una exploración conjunta. Para ello es necesario buscar las alternativas más convenientes para resolver los naturales conflictos que se presenten en cualquier relación humana (Rodino 2012).

\section{Metodología}

Dado las características del contexto se permiten abordar una investigación de tipo cuantitativa, de alcance descriptivo, no experimental, con un diseño de campo. Pues tal como lo expone Hernández, Fernandez y Baptista (2014), se busca estudiar un fenómeno en su contexto natural, no se controlan variables, y se analizan datos a la luz de las variables estudiadas.

\section{Población y muestra}

La población escogida pertenece al Municipio del Banco Magdalena, específicamente de la Institución educativa Departamental Arcesio Cáliz Amador. La muestra estuvo conformada por 30 estudiantes de los grado $8^{\mathrm{a}}$ y $8 \mathrm{~b}$ jornada la mañana.

\section{Instrumentos de recolección} de la información

Para la recolección de los datos se empleó una entrevista estructurada y también la observación, cuyos datos fueron registrados en un cuaderno de notas.

\section{Procedimiento}

Fase (1): Identificación de la problemática a estudiar.

Fase (2): Definición de la población y muestra.

Fase (3): Definición de las variables de estudio. (Valores sociales, relaciones con los compañeros; convivencia y rol de la escuela). Fase (4): Fundamentación teórica.

Fase (5): Escogencia de las técnicas e instrumentos de recolección de información.

Fase (6): Aplicación de los instrumentos.

Fase (7): Análisis y procesamiento de los datos.

Fase (8): Generación de resultados.

Fase (9): Socialización y discusión de los hallazgos encontrados.

\section{Resultados}

A continuación se presentan lo hallazgos derivados de los instrumentos (entrevista). La aplicación se hizo a 25 estudiantes de Grado $8^{\mathrm{a}}$ y $8 \mathrm{~b}$ de la IETD Arcesio Cáliz AMADOR

Para soportar la aplicación se tuvo en cuenta la siguiente pregunta orientadora. ¿Cuál es la la formación en valores sociales que tiene los estudiantes para la promulgación de la convivencia pacífica que contribuya a la consecución de la paz en la institución Arcesio Cáliz Amador?

Variable 1: Valores sociales: En el análisis de los resultados de las entrevistas realizadas a los estudiantes, y de los aspectos que estos manifiestan en torno al interrogante ¿Sabe usted que son los valores sociales? El $63 \%$ de los encuestados respondió que si los conoce, el $37 \%$ restante manifestó que no sabía que es. 
Variable 2: Relaciones con los compañeros: En el análisis de los resultados al siguiente ¿Cómo es su relación con los compañeros de clases? se pudo comprobar que el $58 \%$ de encuestados admitió que en ocasiones las relaciones con sus pares se hacen tensas por pequeñas diferencias y falta de tolerancia, el $42 \%$ de los estudiante sostiene que mantiene relaciones armónicas con sus compañeros.

Variable 3: Convivencia: Las respuestas dadas por los estudiantes sobre el interrogante ¿Ha participado usted en actividades de convivencia? El 95\% respondió que sí, y el 5\% que no. Los entrevistados reconocen que en la institución se realizan actividades que redundan en mejorar las relaciones de convivencia en la Institución Educativa.

Variable 4: El papel de la escuela ante la intolerancia: Los entrevistados respondieron al interrogante ¿Cuál cree usted que debe ser el papel de la escuela ante situaciones de intolerancia en la comunidad educativa? Ante este interrogante los estudiantes respondieron de diversas maneras el 45\% formar agentes de paz, 20\% establecer mecanismos de dialogo, $15 \%$ ser mediadora, $20 \%$ establecer normas y castigos ejemplares

\section{Conclusiones}

Inicialmente el grupo de estudiantes no tenían claro el concepto de valores sociales, primero, porque aunque sabían de la tolerancia y el respeto entre otros, no los reconocían como valores sociales, tenían la concepción que en la IE solo se va a recibir clases para la generación de conocimiento. Se implementaron entonces actividades que fomentaran la autoconciencia y permitieran a los estudiantes convivir pacíficamente en la institución.

Para los docentes, liderar este tipo de investigaciones no solo les permite identificar y darle solución a la problemáticas, sino que además le permiten la consolidación de nuevas estrategias pedagógicas promoviendo la integración curricular de las áreas, intensifican metodologías de trabajo abiertas y contribuyen a la excelencia académica tras el diseño de tareas que son problemáticas que han estado desde siempre y que tienen mayor acogida por los estudiantes desde que se incentive y lidere por parte de ellos. Además, la trasmisión de conocimiento se da en doble vía, el docente aprende mientras enseña, y el estudiante se empodera mientras lo lleva a la práctica.

Finalmente, es importante destacar que, aunque los estudiantes en ocasiones en sus relaciones tengas diferencias y algunas veces conflictos tienen el deseo de convivir pacíficamente y están dispuestos a participar en el desarrollo de las actividades para lograrlo.No obstante, se necesitan en primera instancia, un proceso de motivación en donde los estudiantes participen activamente en nuevas actividades que inviten a la reflexión y a formación en valores para incentivar la convivencia pacífica, conozcan de la importancia de ello y sobre todo cuales son los beneficios que esto a nivel educativo puede generar.

\section{Referencias}

Alvarez, S. (2011). Estrategias de comunicación para padres con hijos adolescentes. (Tesis de Maestría). UADY, Mérida.

Araque, F. y Suárez, O. (2017). Reflexiones teóricas y legales del adulto mayor y la discapacidad en Colombia. Juridicas CUC, 13(1). 97-120. http://dx.doi.org/10.17981/juridcuc.13.1.2017.5

Arrieta, M. (2016). Los masones en el mundo: Geopolítica masónica. La historia de los hijos de la luz. Córdoba: Almuzara.

Avendaño, I., Cortés, O. y Guerrero, H. (2015) Competencias sociales y tecnologías de la información y la comunicación como factores asociados al desempeño en estudiantes de básica primaria con experiencia de desplazamiento forzado. Diversitas: Perspectivas en Psicología, 11(1). 13-36.

Casadiego, J., Martínez, C., Riatiga, A. y Lopez, E. (2015). Habilidades de comunicación asertiva como estrategia en la resolución de conflictos familiares que permite contribuir al desarrollo humano integral en la familia. (Tesis de Pregrado). UNAD. 
Clemente, R. (2015). Relaciones interpersonales y desarrollo humano. Recuperado de http://repositori.uji.es/xmlui/ bitstream/handle/10234/135245/1516 es.pdf;jsessionid=F3B592060FFBB7F 81734E853EB356E8D?sequence $=2$

Cruz, E. (2016). El ciclo de protesta 20102016 en Colombia. Una explicación. Jurídicas CUC, 12(1), 31-62. http://dx.doi. org/10.17981/juridcuc.12.1.2016.3.

Delgado, R. (2010). Relaciones interpersonales en la adolescencia: Implementación de un programa de entrenamiento en asertividad y habilidades sociales para adolescentes de $1^{\circ}$ y $2^{\circ}$ de la ESO. (Tesis de Pregrado). Universidad de Granada, Granada.

Hernández, G., Melendez, M. y Chumaceiro, H. (2017). Cultura democrática, paz y convivencia social en Colombia. Abordaje desde una "Escuela para ciudadanos". En, Conflictos y posconflictos. Pasado y presente en América Latina y el Caribe, caso Colombia-Colección investigación. Cabimas: UNERMB.

Herrera, B., Certain, R. y Calderón, M (2014). La norma hipotética fundamental desde el Paradigma Interpretativo. Justicia Juris, 10(2), 17-27.

Lera, M. (2003). Las relaciones personales en los centros educativos. Recuperado de http://www.psicoeducacion.eu/psicoeducacion/bullying/orientacion/relacionespersonales.pdf

Llorent, V. y Lopez, A. (2012). Estudio de las relaciones interpersonales en aulas con alumnos "diferentes". Campo abierto. 31(2). 87-109.

López, V. (2014). Convivencia Escolar. Apuntes, 4(29). 1-18.

Ministerio de Educación Nacional. (2012). Una propuesta pedagógica para la educación de la primera infancia. Recuperado de http://www.colombiaaprende. edu.co/html/familia/1597/articles-310603_docu2.pdf
Mujica, C. (2013). Propuesta de solución de conflictos entre padres e hijos adolescentes. Recuperado de http://vinculando.org/ padres_e_hijos_familia/solucion-de-conflictos-que-vivencian-los-padres-con-sus-hijosadolescentes.html

Ochoa, R. (2015). Comportamiento Electoral de los Colombianos durante las elecciones presidenciales del año 2010. Jurídicas CUC, 11(1), 209-220. doi: http://dx.doi.org/10.17981/ juridcuc.11.1.2015.9

Oliva, A. (2006). Relaciones familiares y desarrollo adolescente. Anuario de Psicología. 37(3). 209-223.

Pantoja, A. (2005). La gestión de conflictos en el aula. Factores determinantes y propuestas de intervención. Recuperado de https:// www4.ujaen.es/ apantoja/mis_libros/gestion_confli_05.pdf

Rodino, A. (2012). Programa Interamericano sobre Educación en Valores y Prácticas Democráticas. Educación y cultura, 2. Recuperado de http://portal.oas.org/LinkClick.aspx ?fileticket=AMoO5\%2BVNrZ8\%3D\&tab id $=1234$

Sánchez, F. (2017). Conflictos entre padres e hijos: el problema de la disciplina. Adolescere, 5(1).

UNESCO. (2011). Clima, conflictos y violencia en la escuela. Recuperado de https://www. unicef.org/argentina/spanish/clima_conflicto_violencia_escuelas.pdf

Valdarrosa, A. (s.f). Guía familiar solución de problemas en la adolescencia. Recuperado de http://www.edu.xunta.gal/centros/iespontecaldelas/system/files/GUIA\%20ANPA\%20 RESOLUCION\%20DE\%20 CONFLIC TOS\%20CON\%20ILUSTRACIONES.pdf

Vásquez, M. (2010). Condiciones ambientales en la escuela. Recuperado de http://www.consu$\mathrm{m}$ e r . e s/ w e b/e s/e d u c a c i o n / e s c olar/2010/10/22/196660.php

Velazco y Mosquera. (2010). Manual de Estrategias didácticas. Recuperado de http://comisioniberoamericana.org/gallery/ manual-estrategias-didacticas.pdf 